\title{
Article \\ Minimal systems of binomial generators for the ideals of certain monomial curves
}

\author{
Manuel B. Branco ${ }^{1}$ (D), Isabel Colaço ${ }^{2}$ and Ignacio Ojeda I $^{3}$ \\ 1 Departamento de Matemáticas, Universidade de Évora, 7000-671 Évora, Portugal; mbb@uevora.pt \\ 2 Departamento de Matemática e Ciências Físicas, Instituto Politécnico de Beja, 7800-295 Beja, Portugal; \\ isabel.colaco@ipbeja.pt \\ 3 Departamento de Matemáticas, Universidad de Extremadura, 06071 Badajoz, Spain; ojedamc@unex.es \\ * Correspondence: ojedamc@unex.es
}

\begin{abstract}
Let $a, b$ and $n>1$ be three positive integers such that $a$ and $\sum_{j=0}^{n-1} b^{j}$ are relatively prime. In this paper, we prove that the toric ideal $I$ associated to the submonoid of $\mathbb{N}$ generated by $\left\{\sum_{j=0}^{n-1} b^{j}\right\} \cup$ $\left\{\sum_{j=0}^{n-1} b^{j}+a \sum_{j=0}^{i-2} b^{j} \mid i=2, \ldots, n\right\}$ is determinantal. Moreover, we prove that for $n>3$, the ideal $I$ has a unique minimal system of generators if and only if $a<b-1$.
\end{abstract}

Keywords: Binomial ideal, semigroup ideal, minimal system of generators, determinantal ideal, Gröbner basis, indispensability.

MSC: Primary: 13P10, 20M14, secondary: 52B20

\section{Introduction}

Let be a field and let $\mathcal{A}=\left\{a_{1}, \ldots, a_{n}\right\}$ be a set of positive integers. It is well-known that the kernel of the -algebra homomorphism

$$
\varphi_{\mathcal{A}}:\left[x_{1}, \ldots, x_{n}\right] \rightarrow\left[t^{a_{1}}, \ldots, t^{a_{n}}\right] ; x_{i} \mapsto t^{a_{i}}, i=1, \ldots, n,
$$

where $x_{1}, \ldots, x_{n}$ and $t$ are indeterminates, is a binomial ideal (see [6] or [10] for a more recent reference). Clearly, $\operatorname{ker}\left(\varphi_{\mathcal{A}}\right)$ is the defining ideal of a monomial curve.

Let $b$ be a positive integer and set $r_{b}(\ell)$ for the $\ell$-th repunit number in base $b$, that is,

$$
r_{b}(\ell)=\sum_{j=0}^{\ell-1} b^{j}
$$

By convention, $r_{b}(0)=0$.

The main result in this paper is the explicit determination of a minimal system of binomial generators of $I:=\operatorname{ker} \varphi_{\mathcal{A}}$ for

$$
\mathcal{A}=\left\{a_{i}:=r_{b}(n)+a r_{b}(i-1) \mid i=1, \ldots, n\right\},
$$

where $a$ and $n>1$ are positive integers. We prove that $I$ is minimally generated by the $2 \times 2$ minors of the matrix

$$
X:=\left(\begin{array}{cccc}
x_{1}^{b} & \ldots & x_{n-1}^{b} & x_{n}^{b} \\
x_{2} & \ldots & x_{n} & x_{1}^{a+1}
\end{array}\right),
$$

provided that $\operatorname{gcd}\left(a_{1}, \ldots, a_{n}\right)=\operatorname{gcd}\left(a, r_{b}(n)\right)$ is equal to 1 . In this case, as an immediate consequence, we have that the so-called binomial arithmetical rank of $I$ (see, e.g. [8]) is equal to $\left(\begin{array}{l}n \\ 2\end{array}\right)$. 
Furthermore, we obtain that $2 \times 2$-minors of $X$ forms a minimal Gröbner basis with respect to a family of $\mathcal{A}$-graded reverse lexicographical term orders on $\left[x_{1}, \ldots, x_{n}\right]$ (Theorem 1 ) and, applying [12, Corollary 14], we conclude that for $n>3$, the ideal $I$ has a unique minimal system of generators if and only if and $a<b-1$ (Corollary 2).

The submonoids of $\mathbb{N}$ generated by $\mathcal{A}$ are studied in detail in [3] as a generalization of the numerical semigroups introduced by D. Torrão et al. in [13] and [14]; in this context, Corollary 2 provides a minimal presentation of the submonoid of $\mathbb{N}$ generated by $a_{1}, \ldots, a_{n}$, providing an original result not considered in Torrão's PhD thesis.

To achieve our main result (Theorem 1 ), we first compute the ideal $J$ of the projective monomial curve defined by the kernel of the -algebra homomorphism

$$
\left[x_{1}, \ldots, x_{n}\right] \rightarrow\left[t^{r_{b}(0)} s, \ldots, t^{r_{b}(n-1)} s\right] ; \quad x_{i} \mapsto t^{r_{b}(i-1)} s, i=1, \ldots, n,
$$

where $s$ is also an indeterminate. This intermediate result (Proposition 1) has its own interest, since it exhibits another family of semigroup ideals that are determinantal and have unique minimal system of binomial generators (Corollary 1).

Throughout the paper, we keep the notation established in this introduction. Moreover, since the case $n=2$ is trivial and the case $n=3$ is well known for any $a_{1}, a_{2}$ and $a_{3}$ (see [6]), we suppose that $n>3$ whenever necessary.

\section{Gröbner bases and minimal generators for $J$}

In the following, we write $a_{n+k}:=r_{b}(n)+a r_{b}(n+k-1)$ for every $k \geq 1$. Observe that $a_{n+1}=$ $(1+a) r_{b}(n)$.

Lemma 1. For each pair of positive integers $j$ and $k$, it holds that

$$
b a_{j}+a_{j+k}=b a_{j+k-1}+a_{j+1} .
$$

Proof. Since $a_{j+k}=a_{1}+a r_{b}(j+k-1)=a_{1}+a\left(r_{b}(j-1)+b^{j-1} r_{b}(k)\right)=a_{j}+a b^{j-1} r_{b}(k)$, we conclude that

$$
\begin{aligned}
b a_{j}+a_{j+k} & =b a_{j}+a_{j}+a b^{j-1} r_{b}(k)= \\
& =b a_{j}+a_{j}+a b^{j-1}\left(b r_{b}(k-1)+1\right)= \\
& =b\left(a_{j}+a b^{j-1} r_{b}(k-1)\right)+a_{j}+a b^{j-1}= \\
& =b a_{j+k-1}+\left(a_{1}+a r_{b}(j-1)\right)+a b^{j-1}= \\
& =b a_{j+k-1}+a_{j+1},
\end{aligned}
$$

as claimed.

Let $\prec_{i}$ be the term order on $\left[x_{1}, \ldots, x_{n}\right]$ defined by the following matrix

$$
M:=\left(\begin{array}{ccc|c|ccc}
a_{1} & \ldots & a_{i} & a_{i+1} & a_{i+2} & \ldots & a_{n} \\
\hline 0 & & -1 & 0 & 0 & \ldots & 0 \\
& . & & \vdots & \vdots & & \vdots \\
-1 & & 0 & 0 & 0 & \ldots & 0 \\
\hline 0 & \ldots & 0 & 0 & 0 & & -1 \\
\vdots & & \vdots & \vdots & & . . & \\
0 & & 0 & 0 & -1 & & 0
\end{array}\right) .
$$


We observe that $\prec_{i}$ is the $\mathcal{A}$-graded reverse lexicographical term order on $\left[x_{1}, \ldots, x_{n}\right]$ induced by $x_{i} \prec_{i} x_{i-1} \prec_{i} \ldots \prec_{i} x_{1} \prec_{i} x_{n} \prec_{i} \ldots \prec_{i} x_{i+1}$; in particular, $x_{i}$ is the smallest variable for $\prec_{i}$.

Lemma 2. If $j \in\{1, \ldots, n-2\}$ and $k \in\{j+1, \ldots, n-1\}$, then

$$
x_{j}^{b} x_{k+1} \prec_{i} x_{j+1} x_{k}^{b}
$$

if and only if $i \leq j$ or $k+1 \leq i$.

Proof. By Lemma 1, $b a_{j}+a_{k+1}=a_{j+1}+b a_{k}$, so we just need to decide what the variable $x_{j}, x_{j+1}, x_{k}$ or $x_{k+1}$ is cheapest for the order defined by the last $n-1$ rows of $M$. Since $j<j+1 \leq k<k+1$, according to the definition of $\prec_{i}$, the variable $x_{k+1}$ is cheaper than the other three when $j \leq i$ or $k+1 \leq i$; thus, $x_{j}^{b} x_{k+1} \prec_{i} x_{j+1} x_{k}^{b}$ in these cases. Conversely, if $j+1 \leq i \leq k$, then either $x_{k}$ or $x_{j+1}$ is cheaper than the others if $k=i$ or $k \neq i$, respectively. Therefore $x_{j}^{b} x_{k+1} \succ_{i} x_{j+1} x_{k}^{b}$ when $j+1 \leq i \leq k$, and we are done.

Let $I_{2}(Y)$ be the ideal of $\left[x_{1}, \ldots, x_{n}\right]$ generated by the $2 \times 2-$ minors of

$$
Y:=\left(\begin{array}{cccc}
x_{1}^{b} & x_{2}^{b} & \ldots & x_{n-1}^{b} \\
x_{2} & x_{3} & \ldots & x_{n}
\end{array}\right)
$$

Let $\mathcal{G}_{1}^{(i)}, \mathcal{G}_{2}^{(i)}$ and $\mathcal{G}_{3}^{(i)}$ be defined as follows

$$
\begin{gathered}
\mathcal{G}_{1}^{(i)}=\left\{\underline{x_{j+1} x_{k}^{b}}-x_{j}^{b} x_{k+1} \mid j \in\{i, \ldots, n-2\}, k \in\{j+1, \ldots, n-1\}\right\}, \\
\mathcal{G}_{2}^{(i)}=\left\{\underline{x_{j+1} x_{k}^{b}}-x_{j}^{b} x_{k+1} \mid j \in\{1, \ldots, i-2\}, k \in\{j+1, \ldots, i-1\}\right\}, \\
\mathcal{G}_{3}^{(i)}=\left\{\underline{x_{j}^{b} x_{k+1}}-x_{j+1} x_{k}^{b} \mid j \in\{1, \ldots, i-1\}, k \in\{i, \ldots, n-1\}\right\}
\end{gathered}
$$

and let $\mathcal{G}_{Y}^{(i)}$ be equal to $\mathcal{G}_{1}^{(i)} \cup \mathcal{G}_{2}^{(i)} \cup \mathcal{G}_{3}^{(i)}$.

Notice that, by Lemma 2, the underlined monomials are the leading terms with respect to $\prec_{i}$ of the corresponding binomials.

Proposition 1. With the above notation, the set $\mathcal{G}_{Y}^{(i)}$ is the reduced Gröbner basis of $I_{2}(Y)$ with respect to $\prec_{i}$. In particular, the cardinality of $\mathcal{G}_{Y}^{(i)}$ is $\left(\begin{array}{c}n-1 \\ 2\end{array}\right)$.

Proof. First, let us see that $\mathcal{G}_{Y}^{(i)}$ is a Gröbner basis. By the Buchberger's Criterion (see, e.g. [11, Theorem 3.3]), it suffices to verify that each S-pair of elements in $\mathcal{G}_{Y}^{(i)}$ can be reduced to zero by $\mathcal{G}_{Y}^{(i)}$ using the division algorithm. To do this we distinguish several cases:

- Let $f \in \mathcal{G}_{1}^{(i)}$, that is to say, $f=x_{j+1} x_{k}^{b}-x_{j}^{b} x_{k+1}$, for some $j \in\{i, \ldots, n-2\}$ and $k \in\{j+1, \ldots, n-$ $1\}$.

○ Let $g=x_{l+1} x_{m}^{b}-x_{l}^{b} x_{m+1} \in \mathcal{G}_{1}^{(i)}$. If $\operatorname{gcd}\left(x_{j+1} x_{k}^{b}, x_{l+1} x_{m}^{b}\right)=1$, then $S(f, g)$ reduces to zero with respect to $\{f, g\} \subset \mathcal{G}_{Y}^{(i)}$. Otherwise, $j=l, j+1=m, k=l+1$ or $k=m$. If $j=l$ then $S(f, g)=x_{m}^{b}\left(-x_{j}^{b} x_{k+1}\right)-x_{k}^{b}\left(-x_{j}^{b} x_{m+1}\right)=x_{j}^{b}\left(x_{k}^{b} x_{m+1}-x_{m}^{b} x_{k+1}\right)$ reduces to zero with respect to $\mathcal{G}_{Y}^{(i)}$. If $j+1=m$, then

$$
S(f, g)=x_{l+1} x_{j+1}^{b-1}\left(-x_{j}^{b} x_{k+1}\right)-x_{k}^{b}\left(-x_{l}^{b} x_{j+2}\right) .
$$


Now, since $i \leq j<j+1 \leq k<k+1$ and $i \leq l<l+1 \leq m=j+1<j+2$, the leading term of $S(f, g)$ with respect to $\prec_{i}$ is $x_{k}^{b} x_{l}^{b} x_{j+2}$. Then $S(f, g)=x_{l}^{b}\left(x_{k}^{b} x_{j+2}-\right.$ $\left.x_{j+1}^{b} x_{k+1}\right)+x_{j+1}^{b-1} x_{k+1}\left(x_{l}^{b} x_{j+1}-x_{l+1} x_{j}^{b}\right)$ reduces to zero with respect to $\mathcal{G}_{Y}^{(i)}$. By symmetry, the case $k=l+1$ is completely similar to the latter one. Finally, if $k=m$, then $S(f, g)=-x_{l+1}\left(-x_{j}^{b} x_{k+1}\right)-x_{j+1}\left(-x_{l}^{b} x_{k+1}\right)=x_{k+1}\left(x_{j}^{b} x_{l+1}-x_{j+1} x_{l}^{b}\right)$ reduces to zero with respect to $\mathcal{G}_{Y}^{(i)}$.

○ Let $g=x_{l+1} x_{m}^{b}-x_{l}^{b} x_{m+1} \in \mathcal{G}_{2}^{(i)}$. If $\operatorname{gcd}\left(x_{j+1} x_{k}^{b}, x_{l+1} x_{m}^{b}\right)=1$, then $S(f, g)$ reduces to zero with respect to $\{f, g\} \subset \mathcal{G}_{Y}^{(i)}$. Otherwise, $j=l, j+1=m, k=l+1$ or $k=m$. First of all, we observe that the cases $j=l$ and $k=m$ produce the same S-polynomial as in the corresponding case for $g \in \mathcal{G}_{1}^{(i)}$; so, we just focus on the cases $j+1=m$ and $k=l+1$. If $j+1=m$, then $i \leq j<j+1 \leq k<k+1$ and $l<l+1 \leq m=j+1<j+2=m-1 \leq i$, therefore $i<j+1=m<i$, a contradiction. Finally, if $k=l+1$, then $i \leq j<j+1 \leq k<$ $k+1$ and $l<l+1=k \leq m<m+1 \leq i$, so $i<k=l+1<i$, a contradiction again.

○ Let $g=x_{l}^{b} x_{m+1}-x_{l+1} x_{m}^{b} \in \mathcal{G}_{3}^{(i)}$. If $\operatorname{gcd}\left(x_{j+1} x_{k}^{b}, x_{l}^{b} x_{m+1}\right)=1$, then $S(f, g)$ reduces to zero with respect to $\{f, g\} \subset \mathcal{G}_{Y}^{(i)}$. Otherwise, $j+1=l, j=m, k=l$ or $k=m+1$. If $j+1=l$, then $i \leq j<j+1=l \leq k<k+1$ and $l \leq i-1$; so $i<j+1=l \leq i-1$, a contradiction. If $j=m$ (or $k=l$, respectively) then $S(f, g)=x_{m}^{b}\left(x_{k}^{b} x_{l+1}-x_{l}^{b} x_{k+1}\right)$ (or $S(f, g)=x_{k+1}\left(x_{j+1} x_{m}^{b}-x_{j}^{b} x_{m+1}\right)$, respectively) reduces to zero with respect to $\mathcal{G}_{Y}^{(i)}$. Finally, if $k=m+1$, then

$$
S(f, g)=x_{l}^{b}\left(-x_{j}^{b} x_{m+2}\right)-x_{j+1} x_{m+1}^{b-1}\left(-x_{l+1} x_{m}^{b}\right) .
$$

Now, since $i \leq j<j+1 \leq k=m+1<k+1, l \leq i-1$ and $i \leq m$, then $x_{l+1}$ or $x_{m-1}$ is cheaper than the others for the order induced by the last $n-1$ rows of the matrix $M$, therefore leading term of $S(f, g)$ is $x_{l}^{b} x_{j}^{b} x_{k+1}$ and thus, $S(f, g)=-x_{j}^{b}\left(\underline{x_{l}^{b} x_{m+2}}-x_{l+1} x_{m+1}^{b}\right)-$ $x_{l+1} x_{m+1}^{b-1}\left(x_{j}^{b} x_{m+1}-x_{j+1} x_{m}^{b}\right)$ reduces to zero with respect to $\mathcal{G}_{Y}^{(i)}$.

- Let $f \in \mathcal{G}_{2}^{(i)}$, that is to say, $f=x_{j+1} x_{k}^{b}-x_{j}^{b} x_{k+1}$, for some $j \in\{1, \ldots, i-2\}$ and $k \in\{j+1, \ldots, i-$ $1\}$.

○ Let $g=x_{l+1} x_{m}^{b}-x_{l}^{b} x_{m+1} \in \mathcal{G}_{2}^{(i)}$. If $\operatorname{gcd}\left(x_{j+1} x_{k}^{b}, x_{l+1} x_{m}^{b}\right)=1$, then $S(f, g)$ reduces to zero with respect to $\{f, g\} \subset \mathcal{G}_{Y}^{(i)}$. Otherwise, $j=l, j+1=m, k=l+1$ or $k=m$. If $j=l$ (or $k=m$, respectively), then $S(f, g)=x_{j}^{b}\left(x_{k}^{b} x_{m+1}-x_{k+1} x_{m}^{b}\right)$ (or $S(f, g)=$ $x_{k+1}\left(x_{l+1} x_{j}^{b}-x_{l}^{b} x_{j+1}\right)$, respectively) reduces to zero with respect to $\mathcal{G}_{Y}^{(i)}$. If $j+1=m$, then

$$
S(f, g)=x_{l+1} x_{m}^{b-1}\left(-x_{m-1}^{b} x_{k+1}\right)-x_{k}^{b}\left(-x_{l}^{b} x_{m+1}\right)
$$

and, since $l+1 \leq m=j+1 \leq k \leq i-1$, the leading term of $S(f, g)$ is $x_{l+1} x_{m}^{b-1} x_{m-1}^{b} x_{k+1}$. Thus, $S(f, g)=-x_{m}^{b-1} x_{k+1}\left(\underline{x_{l+1} x_{m-1}^{b}}-x_{l}^{b} x_{m}\right)+x_{l}^{b}\left(x_{k}^{b} x_{m+1}-x_{m}^{b} x_{k+1}\right)$ reduces to zero with respect to $\mathcal{G}_{Y}^{(i)}$; observe that $\overline{l<l+1} \leq m$ implies that the leading term of $x_{l+1} x_{m-1}^{b}-x_{l}^{b} x_{m}$ is actually $x_{l+1} x_{m-1}^{b}$. Finally, by symmetry, the case $k=l+1$ is completely similar to the latter one.

。 Let $g=x_{l}^{b} x_{m+1}-x_{l+1} x_{m}^{b} \in \mathcal{G}_{3}^{(i)}$. If $\operatorname{gcd}\left(x_{j+1} x_{k}^{b}, x_{l}^{b} x_{m+1}\right)=1$, then $S(f, g)$ reduces to zero with respect to $\{f, g\} \subset \mathcal{G}_{Y}^{(i)}$. Otherwise, $j+1=l, j=m, k=l$ or $k=m+1$. If $j+1=l$, then

$$
S(f, g)=x_{l}^{b-1} x_{m+1}\left(-x_{l-1}^{b} x_{k+1}\right)-x_{k}^{b}\left(-x_{l+1} x_{m}^{b}\right) .
$$


Furthermore, since $l=j+1 \leq k \leq i-1$ and $l \leq i-1<i \leq m<m+1$, we have that the leading term is $x_{l}^{b-1} x_{m+1} x_{l-1}^{b} x_{k+1}$ if $k=l$ and $x_{k}^{b} x_{l+1} x_{m}^{b}$ otherwise. In ther first case, $S(f, g)=x_{m+1} x_{k-1}^{b}+x_{k} x_{m}^{b}$ reduces to zero with respecto to $\mathcal{G}_{Y}^{(i)}$. In the second case, $S(f, g)=x_{m}^{b}\left(x_{k}^{b} x_{l+1}-x_{k+1} x_{l}^{b}\right)+x_{l}^{b-1} x_{k+1}\left(x_{m}^{b} x_{l}-x_{m+1} x_{l-1}^{b}\right)$ reduces to zero with respect to $\mathcal{G}_{Y}^{(i)}$. If $\overline{j=m}$ (or $k=l$, respectively) then $S(f, g)=x_{m}^{b}\left(x_{k}^{b} x_{l+1}-x_{l}^{b} x_{k+1}\right)$ (or $S(f, g)=x_{k+1}\left(x_{j+1} x_{m}^{b}-x_{j}^{b} x_{m+1}\right)$, respectively) reduces to zero with respect to $\mathcal{G}_{Y}^{(i)}$. Finally, if $k=m+1$, then $j+1 \leq k=m+1 \leq i-1, l \leq i-1$ and $i \leq m$; so, $m+1<i \leq m$, a contradiction.

- Let $f \in \mathcal{G}_{3}^{(i)}$, that is to say, $f=x_{j}^{b} x_{k+1}-x_{j+1} x_{k^{\prime}}^{b}$ for some $j \in\{1, \ldots, i-1\}$ and $k \in\{i, \ldots, n-1\}$.

○ Let $g=x_{l}^{b} x_{m+1}-x_{l+1} x_{m}^{b} \in \mathcal{G}_{3}^{(i)}$. If $\operatorname{gcd}\left(x_{j}^{b} x_{k+1}, x_{l}^{b} x_{m+1}\right)=1$, then $S(f, g)$ reduces to zero with respect to $\{f, g\} \subset \mathcal{G}_{Y}^{(i)}$. Otherwise, $j=l, j=m+1, k+1=l$ or $k=m$. Since $j \leq i-1<i \leq k$ and $l \leq i-1<i \leq m$, the cases $j=m+1$ and $k+1=m$ cannot occur. If $j=l$ (or $k=m$, respectively), then $S(f, g)=x_{l+1}\left(x_{k+1} x_{m}^{b}-x_{m+1} x_{k}^{b}\right)$ (or $S(f, g)=x_{m}^{b}\left(x_{l}^{b} x_{j+1}-x_{j}^{b} x_{l+1}\right)$, respectively) reduces to zero with respect $\mathcal{G}_{Y}^{(i)}$.

Once we know that $\mathcal{G}_{Y}^{(i)}$ is Gröbner basis, it is immediate to see that it is reduced since the leading term of $f \in \mathcal{G}_{Y}^{(i)}$ does not divide any other monomial that appears in a binomial of $\mathcal{G}_{Y}^{(i)} \backslash\{f\}$.

It remains to prove that $\mathcal{G}_{Y}^{(i)}$ generates $I_{2}(Y)$. Clearly, $\mathcal{G}_{Y}^{(i)}$ is contained in the set of $2 \times 2-$ minors of $Y$. Moreover, since the cardinality of $\mathcal{G}_{1}, \mathcal{G}_{2}$ and $\mathcal{G}_{3}$ are

$$
\begin{gathered}
((n-1)-i)+((n-1)-i-1)+\ldots+1=\left(\begin{array}{c}
n-i \\
2
\end{array}\right), \\
((i-1)-1)+((i-1)-2)+\ldots+1=\left(\begin{array}{c}
i-1 \\
2
\end{array}\right)
\end{gathered}
$$

and

$$
(i-1)(n-i),
$$

respectively, we have that the cardinality of $\mathcal{G}_{Y}^{(i)}$ is equal to $\left(\begin{array}{c}n-1 \\ 2\end{array}\right)$ which is the number of $2 \times 2-$ minors of $Y$. Therefore $\mathcal{G}_{Y}^{(i)}$ generates $I_{2}(Y)$ and we are done.

Example 1. We observe that the reduced Gröbner basis, $\mathcal{G}_{Y}^{(i)}$, of $I_{2}(Y)$ with respect to $\prec_{i}$ is not an universal Gröbner basis. For example, if $n=b=5$ and $\prec$ is the term order defined by

$$
\left(\begin{array}{ccccc}
a_{1} & a_{2} & a_{3} & a_{4} & a_{5} \\
0 & 0 & -1 & 0 & 0 \\
0 & 0 & 0 & 0 & -1 \\
0 & 0 & 0 & -1 & 0 \\
0 & -1 & 0 & 0 & 0
\end{array}\right)
$$

then one can check (using, for example, Singular [4]) that the reduced Gröbner basis of the ideal $I_{2}(Y)$ with respect to $\prec$ has eight generators; but $\mathcal{G}_{Y}^{(i)}$ contains $\left(\begin{array}{c}5-1 \\ 2\end{array}\right)=6$ binomials only.

Alternatively, one can see that $\mathcal{G}_{Y}^{(i)}$ is not an universal Gröbner basis of $I_{2}(Y)$ by using [2, Theorem 4.1].

We now consider the $2 \times n$-integer matrix $B$ whose $j-$ th column is

$$
\mathbf{a}_{j}:=\left(\begin{array}{c}
r_{b}(j-1) \\
1
\end{array}\right), \quad j=1, \ldots, n .
$$


Remark 1. Observe that $a_{j}=\left(a, r_{b}(n)\right) \cdot \mathbf{a}_{j}$, for every $j=1, \ldots, n$. (3).

Notice that the semigroup ideal associated to $\left\{\mathbf{a}_{1}, \ldots, \mathbf{a}_{n}\right\}$ is equal to $J$; indeed, $J$ is the kernel of

Corollary 1. The ideal $J$ is minimally generated by the $2 \times 2-$ minors of $Y$. Moreover, J has a unique minimal system of binomial generators.

Proof. Let $I_{2}(Y)$ the ideal generated by the $2 \times 2$-minors of $Y$. Since $b \mathbf{a}_{j}+\mathbf{a}_{k+1}=\mathbf{a}_{j+1}+b \mathbf{a}_{k}$ for every $j$ and $k$, we have that $I_{2}(Y) \subseteq J$.

Conversely, let $C$ be the $(n-2) \times n$-matrix

$$
\left(\begin{array}{cccccccccc}
b & -1 & -b & 1 & 0 & \ldots & 0 & 0 & 0 & 0 \\
0 & b & -1 & -b & 1 & \ldots & 0 & 0 & 0 & 0 \\
0 & 0 & b & -1 & -b & \ldots & 0 & 0 & 0 & 0 \\
0 & 0 & 0 & b & -1 & \ldots & 0 & 0 & 0 & 0 \\
0 & 0 & 0 & 0 & b & \ldots & 0 & 0 & 0 & 0 \\
\vdots & \vdots & \vdots & \vdots & \vdots & \ddots & \vdots & \vdots & \vdots & \vdots \\
0 & 0 & 0 & 0 & 0 & \ldots & b & -1 & -b & 1 \\
0 & 0 & 0 & 0 & 0 & \ldots & 0 & b & -(b+1) & 1
\end{array}\right)
$$

and let $I_{C}$ be the ideal of $\left[x_{1}, \ldots, x_{n}\right]$ generated by

$$
\left\{\mathbf{x}^{\mathbf{u}_{+}}-\mathbf{x}^{\mathbf{u}_{-}} \mid \mathbf{u} \text { is a row of } C\right\},
$$

where $\mathbf{u}_{+}$and $\mathbf{u}_{-}$denote the positive and negative parts of $\mathbf{u}$, respectively. Clearly, $I_{C} \subseteq I_{2}(Y)$.

Now, since the determinant of the submatrix of $C$ consisting in the last $n-2$ columns is 1 , the rows of $C$ generates a rank $n-2$ subgroup $G_{C}$ of $\mathbb{Z}^{n}$ such that $\mathbb{Z}^{n} / G_{C}$ is torsion free. Moreover, since $B C^{\top}=0$, we conclude that the rows of $C$ generate $\operatorname{ker}_{\mathbb{Z}}(B)$. Therefore, by [11, Lemma 7.6],

$$
J=I_{C}:\left(\prod_{j} x_{j}\right)^{\infty} \subseteq I_{2}(Y):\left(\prod_{j} x_{j}\right)^{\infty} .
$$

By Proposition 1 and [1, Theorem 3.1], we have that $I_{2}(Y): x_{i}^{\infty}=I_{2}(Y)$ for every $i=1, \ldots, n$. So, $I_{2}(Y):\left(\prod_{j} x_{j}\right)^{\infty}=I_{2}(Y)$ and, consequently, $J \subseteq I_{2}(Y)$ as desired.

Finally, by Proposition 1, we conclude that the $2 \times 2$-minors of $Y$ form a minimal system generators of $J$ and, [12, Corollary 14], we conclude that $J$ has a unique minimal system of binomial generators.

We recall that semigroup ideals minimally generated by a Graver basis have unique minimal system of binomials generators (see [12, Corollary 16]). As Graver bases are in particular universal Gröbner bases (see [16, Proposition 4.11]), by Example 1, we can assure the minimal system of binomial generators of $J$ is not a Graver basis.

\section{Gröbner basis and minimal generators for $I$}

We keep the notation from the Introduction and Section 2 and we set $\mathcal{G}_{4}$ to be equal to

$$
\left\{\underline{x_{1}^{a+1} x_{l}^{b}}-x_{l+1} x_{n}^{b} \mid l=1, \ldots, i-1\right\} \bigcup\left\{\underline{x_{l+1} x_{n}^{b}}-x_{1}^{a+1} x_{l}^{b} \mid l=i, \ldots, n-1\right\},
$$

where the underlined monomials again highlight the leading terms with respect to $\prec_{i}$ of the corresponding binomials.

Let $I_{2}(X)$ be the ideal of $\left[x_{1}, \ldots, x_{n}\right]$ generated by the $2 \times 2-$ minors of the matrix $X$ defined in (2). 
Theorem 1. The set $\mathcal{G}=\mathcal{G}_{Y}^{(i)} \cup \mathcal{G}_{4}^{(i)}$ is a minimal Gröbner basis of $I_{2}(X)$ with respect to $\prec_{i}$. In particular, the cardinality of $\mathcal{G}$ is $\left(\begin{array}{c}n \\ 2\end{array}\right)$.

Proof. Proceeding as in the proof of Proposition 1, we first need to prove that $S(f, g)$ reduces to zero with respect to $\mathcal{G}^{(i)}$, for every $f, g \in \mathcal{G}^{(i)}$. However, since, by Proposition $1, \mathcal{G}_{Y}^{(i)}$ is already a Gröbner basis with respect to $\prec_{i}$ and the leading terms with respect to $\prec_{i}$ of the binomials in $\mathcal{G}_{4}^{(i)}$ are relatively prime, it suffices to prove that $S(f, g)$ reduces to zero with respect to $\mathcal{G}^{(i)}$, for every $f \in \mathcal{G}_{Y}^{(i)}$ and $g \in \mathcal{G}_{4}^{(i)}$. To do this we distinguish three cases:

- $f \in \mathcal{G}_{1}^{(i)}=\left\{x_{j+1} x_{k}^{b}-x_{j}^{b} x_{k+1} \mid j \in\{i, \ldots, n-2\}, k \in\{j+1, \ldots, n-1\}\right\}$. If $j \neq l$ and $k \neq l+1$, then the leading terms of $f$ and $g$ are relatively prime and there is nothing to prove. So, it suffices to consider the cases $j=l$ or $k=l+1$.

○ If $j=l$, then $l \geq i$, otherwise the leading terms of $f$ and $g$ are relatively prime, and $S(f, g)=$ $x_{n}^{b}\left(-x_{l}^{b} x_{k+1}\right)-x_{k}^{b}\left(-x_{1}^{a+1} x_{l}^{b}\right)=-x_{l}^{b}\left(x_{k+1} x_{n}-x_{1}^{a+1} x_{l}^{b}\right)$ reduces to zero with respect to $\mathcal{G}_{4}^{(i)}$. - If $k=l+1$ then $n-2 \geq k-1=l \geq j \geq i$, otherwise the leading terms of $f$ and $g$ are relatively prime, and $S(f, g)=x_{n}^{b}\left(-x_{j}^{b} x_{l+2}\right)-x_{j+1} x_{l+1}^{b-1}\left(-x_{1}^{a+1} x_{l}^{b}\right)=-x_{j}^{b} x_{n}^{b} x_{l+2}+$ $x_{1}^{a+1} x_{j+1} x_{l+1}^{b-1} x_{l}^{b}$. Observe that the leading term of $S(f, g)$ is divisible by the leading term of $h:=\underline{x_{n}^{b} x_{l+2}}-x_{1}^{a+1} x_{l+1}^{b} \in \mathcal{G}_{4}^{(i)}$. So, $S(f, g)=x_{j}^{b} h-x_{j}^{b} x_{1}^{a+1} x_{l+1}^{b}+x_{1}^{a+1} x_{j+1} x_{l+1}^{b-1} x_{l}^{b}=$ $x_{j}^{b} h-x_{1}^{a+1} x_{l+1}^{b-1}\left(x_{j}^{b} x_{l+1}-x_{j+1} x_{l}^{b}\right)$. Now, since $x_{j}^{b} x_{l+1}-x_{j+1} x_{l}^{b} \in \mathcal{G}_{Y}^{(i)}$, we are done.

- $f \in \mathcal{G}_{2}^{(i)}=\left\{x_{j+1} x_{k}^{b}-x_{j}^{b} x_{k+1} \mid j \in\{1, \ldots, i-2\}, k \in\{j+1, \ldots, i-1\}\right\}$. If $j+1 \neq l$ and $k \neq l$, then the leading terms of $f$ and $g$ are relatively prime and there is nothing to prove. So, it suffices to consider the cases $j=l-1$ or $k=l$.

○ If $j+1=l$, then $1 \leq j=l-1<k \leq i-1$, otherwise the leading terms of $f$ and $g$ are relatively prime, and $S(f, g)=x_{1}^{a+1} x_{l}^{b-1}\left(-x_{l-1}^{b} x_{k+1}\right)-x_{k}^{b}\left(-x_{l+1} x_{n}^{b}\right)=$ $x_{k}^{b} x_{l+1} x_{n}^{b}-x_{1}^{a+1} x_{l-1}^{b} x_{l}^{b-1} x_{k+1}$. If $l=k$, then the S-polynomial $S(f, g)=x_{k}^{b} x_{k+1} x_{n}^{b}-$ $x_{1}^{a+1} x_{k-1}^{b} x_{k}^{b-1} x_{k+1}=-x_{k}^{b-1} x_{k+1}\left(x_{1}^{a+1} x_{k-1}^{b}-x_{k} x_{n}^{b}\right)$ reduces to zero with respect to $\mathcal{G}_{4}^{(i)} ;$ otherwise, the leading term of $S\left(\overline{f, g) \text { is } x_{k}^{b}} x_{l+1} x_{n}^{b}\right.$ which is divisible by the leading term of $h:=\underline{x_{k}^{b} x_{l+1}}-x_{k+1} x_{l}^{b} \in \mathcal{G}_{4}^{(i)}$. So, $S(f, g)=x_{n}^{b} h+x_{n}^{b} x_{k+1} x_{l}^{b}-x_{1}^{a+1} x_{l-1}^{b} x_{l}^{b-1} x_{k+1}=$ $x_{n}^{b} h-x_{l}^{b-1} x_{k+1}\left(x_{1}^{a+1} x_{l-1}^{b}-x_{n}^{b} x_{l}\right)$. Now, since $x_{1}^{a+1} x_{l-1}^{b}-x_{n}^{b} x_{l} \in \mathcal{G}_{4}^{(i)}$, we are done.

- If $k=l$, then $1 \leq j<k=l \leq i-1$, otherwise the leading terms of $f$ and $g$ are relatively prime, and $S(f, g)=x_{1}^{a+1}\left(-x_{j}^{b} x_{l+1}\right)-x_{j+1}\left(-x_{l+1} x_{n}^{b}\right)=-x_{l+1}\left(x_{1}^{a+1} x_{j}^{b}-x_{j+1} x_{n}^{b}\right)$. Now, since $x_{1}^{a+1} x_{j}^{b}-x_{j+1} x_{n}^{b} \in \mathcal{G}_{4}^{(i)}$, we are done.

- $f \in \mathcal{G}_{3}^{(i)}=\left\{x_{j}^{b} x_{k+1}-x_{j+1} x_{k}^{b} \mid j \in\{1, \ldots, i-1\}, k \in\{i, \ldots, n-1\}\right\}$. If $j \neq 1, j \neq l, k \neq l$ and $k \neq n-1$, then the leading terms of $f$ and $g$ are relatively prime and there is nothing to prove. So, is suffices so consider the cases $j=1, j=l, k=l$ or $k=n-1$.

- If $j=1$, then, in particular, $l<i$, otherwise the leading terms of $f$ and $g$ are relatively prime. Now, if $a+1 \geq b$, then $S(f, g)=x_{1}^{a+1-b} x_{l}^{b}\left(-x_{2} x_{k}^{b}\right)-x_{k+1} x_{l+1} x_{n}^{b}$ and its leading term is divisible by the leading term of $h:=x_{l}^{b} x_{2}-x_{l+1} x_{1}^{b} \in \mathcal{G}_{2}^{(i)} \cup \mathcal{G}_{3}^{(i)}$; then $S(f, g)=x_{1}^{a+1-b} x_{k}^{b} h-$ $x_{1}^{a+1-b} x_{k}^{b}\left(-x_{l+1} x_{1}^{b}\right)-x_{k+1} x_{l+1} x_{n}^{b}=\overline{x_{1}^{a+1}}-b x_{k}^{b} h-x_{l+1}\left(x_{k+1} x_{n}^{b}-x_{1}^{a+1} x_{k}^{b}\right)$ which reduces to zero with respect to $\mathcal{G}_{2}^{(i)} \cup \mathcal{G}_{3}^{(i)} \cup \mathcal{G}_{4}^{(i)}$. Otherwise, if $a+\overline{1<b}$, then $S(f, g)=\underline{x_{l}^{b}\left(-x_{2} x_{k}^{b}\right)-}$ $x_{1}^{b-a-1} x_{k+1}\left(-x_{l+1} x_{n}^{b}\right)$ and its leading term is divisible by the leading term of $h:=x_{l}^{b} x_{2}-$ $x_{l+1} x_{1}^{b} \in \mathcal{G}_{2}^{(i)} \cup \mathcal{G}_{3}^{(i)}$; then $S(f, g)=x_{k}^{b} h-x_{k}^{b}\left(-x_{l+1} x_{1}^{b}\right)-x_{1}^{b-a-1} x_{k+1}\left(-x_{l+1} x_{n}^{b}\right)=\overline{x_{k}^{b} h}-$ $x_{1}^{b-a-1} x_{l+1}\left(x_{k+1} x_{n}^{b}-x_{1}^{a+1} x_{k}^{b}\right)$ which reduces to zero with respect to $\mathcal{G}_{2}^{(i)} \cup \mathcal{G}_{3}^{(i)} \cup \mathcal{G}_{4}^{(i)}$, too. 
○ If $j=l$, then $1 \leq l \leq i-1$, otherwise the leading terms of $f$ and $g$ are relatively prime, and $S(f, g)=x_{1}^{a+1}\left(-x_{l+1} x_{k}^{b}\right)-x_{k+1}\left(-x_{l+1} x_{n}^{b}\right)=-x_{l+1}\left(x_{k+1} x_{n}^{b}-x_{1}^{a+1} x_{k}^{b}\right)$ which reduces to zero with respect to $\mathcal{G}_{4}^{(i)}$.

$\circ$ If $k=l$, then $i \leq l \leq n-1$, otherwise the leading terms of $f$ and $g$ are relatively prime, and $S(f, g)=x_{n}^{b}\left(-x_{j+1} x_{l}^{b}\right)-x_{j}^{b}\left(-x_{1}^{a+1} x_{l}^{b}\right)=x_{l}^{b}\left(x_{1}^{a+1} x_{j}^{b}-x_{j+1} x_{n}^{b}\right)$ which reduces to zero with respect to $\mathcal{G}_{4}^{(i)}$.

$\circ$ If $k=n-1$, then $1 \leq j<i \leq l<n$, otherwise the leading terms of $f$ and $g$ are relatively prime. In this case, $S(f, g)=x_{l+1} x_{n}^{b-1}\left(-x_{j+1} x_{n-1}^{b}\right)-x_{j}^{b}\left(-x_{1}^{a+1} x_{l}^{b}\right)$ and, since the leading term of $S(f, g)$ is divisible by the leading term of $h:=\overline{x_{1}^{a+1} x_{j}^{b}-x_{j+1}} x_{n}^{b} \in \mathcal{G}_{4}^{(i)}$, we have that $S(f, g)=x_{l}^{b} h-x_{l}^{b}\left(-x_{j+1} x_{n}^{b}\right)+x_{l+1} x_{n}^{b-1}\left(-x_{j+1} x_{n-1}^{b}\right)=x_{l}^{b} h-x_{j+1} x_{n}^{b-1}\left(x_{l+1} x_{n-1}^{b}-x_{l}^{b} x_{n}\right)$, and since $x_{l+1} x_{n-1}^{b}-x_{l}^{b} x_{n}$ belongs to $\mathcal{G}_{1}^{(i)}$, we are done.

Now, since $S(f, g)$ reduces to zero with respect to $\mathcal{G}^{(i)}$ in all the three cases we conclude that $\mathcal{G}^{(i)}$ forms a Gröbner basis.

Once we know that $\mathcal{G}^{(i)}$ is a Gröbner basis, we observe that the leading terms of the binomials in $\mathcal{G}^{(i)}$ are not divisible by the leading term of any other binomial in $\mathcal{G}^{(i)}$ other than itself. That is to say, the Gröbner basis $\mathcal{G}^{(i)}$ is minimal.

Clearly, $\mathcal{G}^{(i)}$ is a subset of $2 \times 2$-minors of $X$. Moreover, its cardinality is equal to the cardinality of $\mathcal{G}_{Y}^{(i)}$, that is $\left(\begin{array}{c}n-1 \\ 2\end{array}\right)$, plus the cardinality, $n-1$, of $\mathcal{G}_{4}^{(i)}$. Therefore, $\mathcal{G}^{(i)}$ has cardinality equal to $\left(\begin{array}{c}n-1 \\ 2\end{array}\right)+(n-1)=\left(\begin{array}{l}n \\ 2\end{array}\right)$ which is the number of $2 \times 2$-minors of $X$. Hence we conclude that $\mathcal{G}^{(i)}$ generates $I_{2}(X)$ and we are done.

Example 2. The minimal Gröbner basis, $\mathcal{G}^{(i)}$, of $I_{2}(X)$ with respect to $\prec_{i}$ is not reduced in general. For example, if $n=4, a=3$ and $b=3$, then one can see (using, e.g. Singular [4]) that the binomial $x_{4}^{4}-x_{1} x_{2}^{4} x_{3}^{2}$ belongs to the Gröbner basis of $I_{2}(X)$ with respect to $\prec_{2}$, however, $x_{4}^{4}-x_{1} x_{2}^{4} x_{3}^{2}$ is not a minor of $X$.

Corollary 2. If $\operatorname{gcd}\left(a, r_{b}(n)\right)=1$, then the ideal I is minimally generated by the $2 \times 2$-minors of $X$. In this case, if $n>3$, then I has a unique minimal system of generators if and only if and $a<b-1$.

Proof. By Theorem 1, to prove the first part of the statement it suffices to see that $I=I_{2}(X)$.

By Lemma 1, we have that $\varphi_{\mathcal{A}}(f)=0$, for every $f \in \mathcal{G}^{(i)}$, where $\varphi_{\mathcal{A}}$ is the -algebra homomorphism define in (1). Therefore $I_{2}(X) \subseteq I$. Conversely, let $L$ be the $(n-1) \times n$-matrix

$$
\left(\begin{array}{cccccccc}
b & -(b+1) & 1 & 0 & \ldots & 0 & 0 & 0 \\
0 & b & -(b+1) & 1 & \ldots & 0 & 0 & 0 \\
\vdots & \vdots & \vdots & \vdots & & \vdots & \vdots & \vdots \\
0 & 0 & 0 & 0 & \ldots & b & -(b+1) & 1 \\
(a+1) & 0 & 0 & 0 & \ldots & 0 & b & -(b+1)
\end{array}\right)
$$

and let $I_{L}$ be the ideal of $\left[x_{1}, \ldots, x_{n}\right]$ generated by

$$
\left\{\mathbf{x}^{\mathbf{u}_{+}-} \mathbf{x}^{\mathbf{u}_{-}} \mid \mathbf{u} \text { is a row of } L\right\} .
$$

Clearly, $I_{L} \subseteq I_{2}(X)$.

On the one hand, a direct computation shows that the set of $(n-1) \times(n-1)-$ minors of $L$ is equal to $\left\{a_{1}, \ldots, a_{n}\right\}$ and therefore, by [16, Lemma 12.2],

$$
I_{L}:\left(\prod_{i=1}^{n} x_{i}\right)^{\infty}=I
$$


if and only if $\operatorname{gcd}\left(a_{1}, \ldots, a_{n}\right)=\operatorname{gcd}\left(a, r_{b}(n)\right)=1$. On the other hand, by Theorem 1 and [1, Theorem 3.1], we have that $I_{2}(X):\left(x_{i}^{\infty}\right)=I_{2}(X)$ for every $i=1, \ldots, n$, that is to say, $I_{2}(X):\left(\prod_{i=1}^{n} x_{i}\right)^{\infty}=$ $I_{2}(X)$. Putting this together we conclude that

$$
I_{2}(X)=I_{2}(X):\left(\prod_{i=1}^{n} x_{i}\right)^{\infty} \supseteq I_{L}:\left(\prod_{i=1}^{n} x_{i}\right)^{\infty}=I,
$$

and thus $I=I_{2}(X)$ as claimed.

To prove the second part of the statement, we observe that, for every $i \neq n$, the non-leading term,

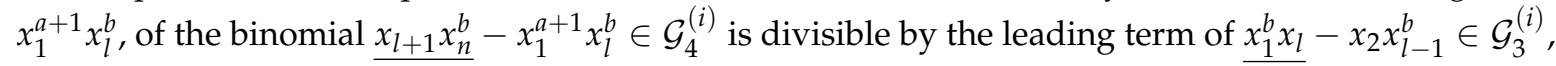
provided that $l \geq 3$ (otherwise no such binomial in $\mathcal{G}_{3}^{(i)}$ exists), if and only $a+1 \geq b$. Now, as these are the only divisibility relationships between the monomials of the binomials in $\mathcal{G}^{(i)}$, and $l \geq 3$ implicitly requires $n>3$, we obtain that for $n>3, \mathcal{G}^{(i)}$ is reduced for every $\prec_{i}$ if and only $a<b-1$, and, by [12, Corollary 14], we conclude that for $n>3, I$ has a unique minimal system of binomial generators if and only if and $a<b-1$.

Notice that the condition $\operatorname{gcd}\left(a, r_{b}(n)\right)=1$ cannot be avoided.

Example 3. Let $n=4, a=3$ and $b=2$. In this case, $a_{1}=r_{b}(4)=15, a_{2}=18, a_{3}=24$ and $a_{4}=36$. Clearly, $\operatorname{gcd}\left(a_{1}, a_{2}, a_{3}, a_{4}\right)=\operatorname{gcd}\left(a, r_{b}(4)\right)=3$. By direct computation, one can check that I is minimally generated by four binomials whereas $I_{2}(X)$ is minimally generated by six binomials. In particular, $I \neq I_{2}(X)$; in fact, one has that $I$ is a minimal prime of $I_{2}(X)$.

The following example shows the minimal system of generators of $I$ for $n=4$.

Example 4. If $n=4$, then the ideal $I \subset\left[x_{1}, x_{2}, x_{3}, x_{4}\right]$ is minimally generated by

$$
x_{2}^{b+1}-x_{1}^{b} x_{3}, x_{1}^{b} x_{4}-x_{2} x_{3}^{b}, x_{3}^{b+1}-x_{2}^{b} x_{4}
$$

and

$$
x_{1}^{a+b+1}-x_{2} x_{4}^{b}, x_{1}^{a+1} x_{2}^{b}-x_{3} x_{4}^{b}, x_{4}^{b+1}-x_{1}^{a+1} x_{3}^{b}
$$

(recall that the first three binomials generates J). In [7], a complete classification of the monomial curves in $\mathbb{A}^{4}()$ having a unique minimal system of generators is given. By [7, Theorem 3.11], one has that I has a unique minimal system of generators if and only if $x_{1}^{a+1} x_{3}^{b}$ is not divisible by $x_{1}^{b} x_{3}$; equivalently $a<b-1$ as we already knew by Corollary 2. Observe that the result on the uniqueness of the system of generators of I can be deduced from [9], too.

We end this paper by observing that, since both $J$ and $I$ are determinantal ideals by Corollaries 1 and 2, respectively, one can conveniently adapt [5, Section 2.1] to compute the minimal free resolution of $I$ and $J$ using the Eagon-Northcott complex. In particular, the - algebras $\left[x_{1}, \ldots, x_{n}\right] / J$ and $\left[x_{1}, \ldots, x_{n}\right] / I$ are Cohen-Macaulay of type $n-2$ and $n-1$, respectively (see [5, Section 2.1] for further details).

Funding: This reserach was partially supported by the Ministerio de Ciencia e Innovación (Spain) /FEDER-UE under grant PGC2018-096446-B-C21, by the Ministerio de Ciencia e Innovación (Spain) /FEDER-UE under grant MTM2017-84890-P, by the Junta de Extremadura(Spain)/FEDER funds, research group FQM-024,

\section{References}

1. Bigatti, A.M.; La Scala, R.; Robbiano, L. Computing toric ideals. J. Symb. Comput. 27 (1999) 351-365.

2. Boocher, A.; Robeva, E. Robust toric ideals. J. Symb. Comput. 68(1) (2015), 254-264.

3. Branco, M.B.; Colaço, I., Ojeda, I. Generalized repunit numerical semigroups (work in progress). 
4. Decker, W.; Greuel, G.-M.; Pfister, G.; Schönemann, H. SiNGULAR 4-2-1 — A computer algebra system for polynomial computations. http:/ / www.singular.uni-kl.de (accessed on 28 October 2021).

5. Gimenez, P.; Sengupta, I.; Srinivasan, H. Minimal free resolutions for certain affine monomial curves. Contemporary Mathematics 555 (2011) 87-95.

6. Herzog, J. Generators and relations of abelian semigroups and semigroup rings. Manuscripta Math. 3 (1970), 175-193.

7. Katsabekis, A., Ojeda, I. An indispensable classification of monomial curves in $\mathbb{A}^{4}()$. Pacific J. Math. 268 (2014), 96-116.

8. Katsabekis, A. Arithmetical rank of binomial ideals. Arch. Math. 109 (2017), 323-334.

9. Katsabekis, A., Thoma, A. Specializations of multigradings and the arithmetical rank of lattice ideals. Comm. Algebra 38 (2010), 1904-1918.

10. Matusevich, L.F., Ojeda, I. Binomial ideals and congruences on $\mathbb{N}^{n}$. In: Greuel, G.M., et al. (eds.) Singularities, Algebraic Geometry, Commutative Algebra, and Related Topics Festschrift for Antonio Campillo on the Occasion of his 65th Birthday, pp. 429-454. Springer, Berlin (2018)

11. Miller, E.; Sturmfels, B. Combinatorial Commutative Algebra. Vol. 227 of Graduate Texts in Mathematics. Springer, New York. 2005.

12. Ojeda, I; Vignerón-Tenorio, A. Indispensable binomials in semigroups ideals. Proc. Amer. Math. Soc. 138 (2010), 4205-4216.

13. Rosales, J.C.; Branco, M.B.; Torrão, D. The Frobenius problem for repunit numerical semigroups, Ramanujan J. 40 (2016), 323-334.

14. Rosales, J.C.; Branco, M.B.; Torrão, D. The Frobenius problem for Mersenne numerical semigroups, Math. Z. 286 (2017), 741-749.

15. Rosales, J.C.; García-Sánchez, P.A. Numerical semigroups. Developments in Mathematics, vol.20, Springer, New York, (2009).

16. Sturmfels, B. Gröbner bases and convex polytopes, AMS University Lecture Series Vol. 8, American Mathematical Society, Providence, RI, 1996. 